\title{
Supergravity Model of Inflation and Explaining IceCube HESE Data via PeV Dark Matter Decay
}

\author{
Girish Kumar Chakravarty, ${ }^{1}$ Najimuddin Khan $\mathbb{D}^{,},{ }^{2}$ and Subhendra Mohanty ${ }^{3}$ \\ ${ }^{1}$ Centre for Theoretical Physics, Jamia Millia Islamia, New Delhi, India \\ ${ }^{2}$ School of Physical Sciences, Indian Association for the Cultivation of Science, Kolkata, India \\ ${ }^{3}$ Theoretical Physics Division, Physical Research Laboratory, Ahmedabad, India
}

Correspondence should be addressed to Najimuddin Khan; khanphysics.123@gmail.com

Received 1 February 2020; Revised 6 June 2020; Accepted 8 July 2020; Published 1 August 2020

Academic Editor: Ricardo G. Felipe

Copyright (c) 2020 Girish Kumar Chakravarty et al. This is an open access article distributed under the Creative Commons Attribution License, which permits unrestricted use, distribution, and reproduction in any medium, provided the original work is properly cited. The publication of this article was funded by SCOAP ${ }^{3}$.

We construct a unified model of inflation and PeV dark matter with an appropriate choice of no-scale Kähler potential, superpotential, and gauge kinetic function in terms of MSSM fields and hidden sector Polonyi field. The model is consistent with the $\mathrm{CMB}$ observations and can explain the PeV neutrino flux observed at IceCube HESE. A Starobinsky-like Higgssneutrino plateau inflation is obtained from the $D$-term SUGRA potential while $F$-term being subdominant during inflation. To get PeV dark matter, SUSY breaking at PeV scale is achieved through Polonyi field. This sets the scale for soft SUSY breaking parameters $m_{0}, m_{1 / 2}, A_{0}$ at the GUT scale in terms of the parameters of the model. The low-energy particle spectrum is obtained by running the RGEs. We show that the $\sim 125 \mathrm{GeV}$ Higgs and the gauge coupling unification can be obtained in this model. The $6 \mathrm{PeV}$ bino-type dark matter is a subdominant fraction $(\sim 11 \%)$ of the relic density, and its decay gives the PeV scale neutrino flux observed at IceCube by appropriately choosing the couplings of the $R$-parity violating operators. Also, we find that there is degeneracy in scalar field parameters $(\gamma, \beta)$ and coupling $\zeta$ value in producing the correct amplitude of CMB power spectrum. However, the value of parameter $\tan (\beta)=1.8$, which is tightly fixed from the requirement of PeV scale SUSY breaking, removes the degeneracy in the values of the scalar field parameters to provide a unique solution for inflation. In this way, it brings the explanation for dark matter, $\mathrm{PeV}$ neutrinos, and inflation within the same framework.

\section{Introduction}

The $125 \mathrm{GeV}$ Higgs boson found at the Large Hadron Collider (LHC) [1-3] completes the spectrum of the standard model but raises the question about what protects the mass of the Higgs at the electroweak scale despite quantum corrections (the gauge hierarchy problem). Supersymmetry [4-6] has been widely accepted as the natural symmetry argument for protecting the Higgs and other scalar masses against radiative corrections and in addition provides the unification of the gauge couplings at the GUT scale and WIMP dark matter. The idea of naturalness in SUSY $[7,8]$ requires that to explain the Higgs mass, loop corrections should not be too large compared to the tree level and should not rely on the cancellation of large corrections from different loop corrections. This puts upper bounds on the masses of squarks, gluons, and gauginos for natural SUSY [8]. The searches for SUSY particles at LHC by ATLAS [9] and CMS [10] at $13 \mathrm{TeV}$ and with $15 \mathrm{f} b^{-1}$ of data do not find the SUSY partners and rule out the simplest models of natural SUSY $[11,12]$. The idea of supersymmetry for explaining the Higgs mass without fine-tuning can be abandoned while still retaining some of the positive features like coupling constant unification and WIMP dark matter in split SUSY [13] scenarios where squarks and gluinos are heavy evading the LHC bounds and the electroweakinos are of the $\mathrm{TeV}$ scale providing the WIMP dark matter and coupling constant unification. With the tight bounds from the direct detection experiments $[14,15]$, the bino-higgsino dark matter of a few $100 \mathrm{GeV}$ is getting increasingly difficult [16-25]. The evidence that there is need for new physics at $\mathrm{PeV}$ scale comes from IceCube's High-Energy 
Starting Events (HESE) observations [26-29] of $\mathrm{PeV}$ energy neutrinos. The nonobservation of neutrinos with deposited energy between 0.4 and $1 \mathrm{PeV}$ and at Glashow resonance energies has called for an extra source of $\mathrm{PeV}$ energy neutrinos, and a popular scenario [30-35] is the decay of $\mathrm{PeV}$ scale dark matter with a sizable branching to neutrinos and with a lifetime of $\sim 10^{15} \mathrm{sec}$ [36] and $\sim 10^{28} \mathrm{sec}$ [37-50]. Leptoquarks have been used to explain IceCube PeV events in Refs. [51-56]. A PeV scale supersymmetry model with gauge coupling unification, light Higgs (with fine-tuning), and $\mathrm{PeV}$ dark matter was introduced in [57, 58]. It was found [39] that in order to obtain the large decay time of $\sim 10^{28} \mathrm{sec}$ as required from the observed IceCube neutrino flux, dimension 6 operators suppressed by the GUT scale had to be introduced in the superpotential.

A different motivation for supersymmetry is its usefulness in inflation. The low upper bound on the tensor-toscalar ratio by Planck [59-61] and BICEP2/Keck Array (BK15) $[62,63]$ rules out the standard particle physics models with quartic and quadratic potentials. One surviving model is the Starobinsky $R+R^{2}$ model which predicts a very low tensor-to-scalar ratio of $\sim 10^{-3}$. It was shown by Ellis et al. [64] that by choosing the Kähler potential of the noscale form one can achieve Starobinsky-type plateau inflation in a simple Wess-Zumino model. The no-scale model of inflation from $F$-term has been constructed in $\mathrm{SO}(10)$ $[65,66], \operatorname{SU}(5)$ [67], NMSSM [68], and MSSM [69, 70] models. Inflation models in supergravity with F-term scalar potential were earlier considered in [71-75] and with a $D$-term scalar potential in [76-79]. The Higgs-sneutrino inflation along the $D$-flat directions in MSSM has also been studied in [80-86].

In this paper, we construct a $D$-term inflation model with no-scale Kähler potential, MSSM superpotential, and an appropriate choice of gauge kinetic function in MSSM fields, which gives a Starobinsky-like Higgs-sneutrino plateau inflation favored by observations. The SUSY breaking scale is a few $\mathrm{PeV}$ which provides a $\mathrm{PeV}$ scale bino as dark matter. A fraction of thermal relic density is obtained by turning on a small $R$-parity violation to give a decaying dark matter whose present density and neutrino flux at IceCube is tuned by choosing the $R$-parity violating couplings. Superysmmetry breaking is achieved by a hidden sector Polonyi field which takes a nonzero vev at the end of inflation. The gravitino mass is a few PeV which sets the SUSY breaking scale. The mSUGRA model has only five free parameters including soft SUSY breaking parameters. These are the common scalar mass $m_{0}$, the common gaugino mass $m_{1 / 2}$, the common trilinear coupling parameter $A_{0}$, the ratio of Higgs field vevs $\tan \beta$, and the sign of mass parameter $\mu$; all are given at the gauge coupling unification scale. The spectra and the couplings of sparticles at the electroweak symmetry breaking scale are generated by renormalization group equations (RGEs) of the above soft breaking masses and the coupling parameters. The sparticle spectrum at the low-energy scale was generated using the publicly available softwares FlexibleSUSY [87, 88], SARAH [89, 90], and SPheno [91, 92] with the mSUGRA input parameter set $m_{0}, m_{1 / 2}, A_{0}$, $\tan \beta$, and $\operatorname{sign}(\mu)$. In our analysis, we have used SARAH to generate model files for mSUGRA and relic density of LSP has been calculated using micrOMEGAs [93, 94].

$R$-parity conserving SUSY models include a stable, massive weakly interacting particle (WIPM) and the lightest supersymmetric particle (LSP), namely, neutralino, which can be considered as a viable dark matter candidate. With the additional very tiny $R$-parity violating (RPV) terms, LSP can decay to SM particles [56, 95-97]; however, it does not change the effective (co)annihilation cross section appreciably in the Boltzmann equation [98-100]. In the context of mSUGRA model, the analysis of neutralino dark matter has been carried out in Refs. [101-113], where SUSY breaking occurs in a hidden sector, which is communicated to the observable sector via gravitational interactions.

The paper is organized as follows. In Section 2, we display the MSSM model and the specific form of a Kähler potential and gauge kinetic function which gives the Starobinsky-like Higgs-sneutrino plateau inflation. The $R$-parity violating terms contribute to the $F$-term potential and to the decay of the dark matter for IceCube. We fix the parameter in the gauge kinetic function and show that a $D$-term inflation model consistent with the CMB observations is obtained. In Section 3, we describe the SUSY breaking mechanism from the Polonyi field and calculate the soft breaking parameters $m_{0}, m_{1 / 2}, A_{0}$ and gravitino mass $m_{3 / 2}$ as a function of parameters of the Polonyi potential. In Section 4, we give the phenomenological consequences of the PeV scale SUSY model. We show that the coupling constant unification can take place and we obtain $\sim 125 \mathrm{GeV}$ Higgs mass by fine-tuning. The relic density of the bino LSP is overdense after thermal decoupling, but due to slow decay from the $R$-parity violation, a fraction of relic density remains in the present epoch. A different $R$-parity operator is responsible for the decay of the PeV dark matter into neutrinos. We fix the parameters of the $R$-parity violating couplings to give the correct flux of the PeV scale neutrino events seen at IceCube. In Section 5, we summarize the main results and give our conclusions.

\section{2. $D$-Term mSUGRA Model of Inflation}

We consider the model with MSSM matter fields and $R$-parity violation and choose a Kähler potential $K\left(\phi_{i}, \phi_{i}^{*}\right.$, $\left.Z, Z^{*}\right)$ and gauge kinetic function $f_{a b}\left(\phi_{i}, Z\right)$ with the aim of getting a plateau inflation favored by observations. We choose $K$ and $f_{a b}$ of the form

$$
\begin{gathered}
K=-3 \ln \left[1-\frac{1}{3}\left(H_{u}^{\dagger} H_{u}+H_{d}^{\dagger} H_{d}+L^{\dagger} L+Q^{\dagger} Q\right.\right. \\
\left.\left.+\tilde{e}_{R}^{*} \tilde{e}_{R}+\tilde{u}_{R}^{*} \tilde{u}_{R}+\tilde{d}_{R}^{*} \tilde{d}_{R}\right)\right]+Z Z^{*}+\frac{\alpha}{2}\left(Z Z^{*}\right)^{2}, \\
f_{a b}=\frac{e^{-\kappa Z}}{1+\zeta H_{u} \cdot H_{d}} \delta_{a b},
\end{gathered}
$$

respectively. And the MSSM superpotential $W\left(\phi_{i}\right)$ 


$$
\begin{aligned}
W= & \mu H_{u} \cdot H_{d}-Y_{d} Q \cdot H_{d} \tilde{d}_{R}+Y_{u} Q \cdot H_{u} \tilde{u}_{R} \\
& -Y_{e} L \cdot H_{d} \tilde{e}_{R}+\mu_{z}^{2} Z+\mu_{z z} Z^{3}
\end{aligned}
$$

where

$$
\begin{gathered}
H_{u}=\left(\begin{array}{l}
\phi_{u}^{+} \\
\phi_{u}^{0}
\end{array}\right), \\
H_{d}=\left(\begin{array}{l}
\phi_{d}^{0} \\
\phi_{d}^{-}
\end{array}\right), \\
L=\left(\begin{array}{l}
\phi_{v} \\
\phi_{e}
\end{array}\right), \\
Q=\left(\begin{array}{l}
u_{L} \\
d_{L}
\end{array}\right),
\end{gathered}
$$

and field $Z$ is the hidden sector Polonyi which is introduced to break supersymmetry. The Polonyi field $Z$ is associated with the fluctuations in the overall size of the compactified dimensions which has to be strongly stabilized at SUSY breaking for the successful implementation of inflation in supergravity. The parameters $\alpha, \kappa, \mu_{z}, \mu_{z z}$, and $\zeta$ are coupling constants of the model to be fixed from SUSY breaking and inflation. The other fields bear their standard meanings. Also, the above potentials are in the $M_{p}=(8 \pi G)^{-1}=1$ unit and shall use the same convention throughout the analysis of this model.

In addition to the superpotential given in equation (3), we also consider the $R$-parity violating interaction terms

$$
W_{\text {int }}=\lambda_{i j k} L_{i} L_{j} e_{R k}^{c}+\lambda_{i j k}^{\prime} L_{i} Q_{j} \mathrm{~d}_{R k}^{c}+\frac{1}{2} \lambda_{i j k}^{\prime} u_{R i}^{c} d_{R j}^{c} d_{R k}^{c},
$$

which will play a role in explaining observed DM relic density and $\mathrm{PeV}$ neutrino flux at IceCube.

In supergravity, the scalar potential depends upon the Kähler function $G\left(\phi_{i}, \phi_{i}^{*}\right)$ given in terms of superpotential $W\left(\phi_{i}\right)$ and Kähler potential $K\left(\phi_{i}, \phi_{i}^{*}\right)$ as $G\left(\phi_{i}, \phi_{i}^{*}\right) \equiv K\left(\phi_{i}\right.$, $\left.\phi_{i}^{*}\right)+\ln W\left(\phi_{i}\right)+\ln W^{*}\left(\phi_{i}^{*}\right)$, where $\phi_{i}$ are the chiral scalar superfields. In $\mathscr{D}=4, \mathcal{N}=1$ supergravity, the total tree-level supergravity scalar potential is given as the sum of F-term and $D$-term potentials which is given by

$$
\begin{aligned}
V_{F} & =e^{G}\left[\frac{\partial G}{\partial \phi^{i}} K_{j *}^{i} \frac{\partial G}{\partial \phi_{j}^{*}}-3\right], \\
V_{D} & =\frac{1}{2}\left[\operatorname{Re} f_{a b}\right]^{-1} D^{a} D^{b},
\end{aligned}
$$

respectively, where $D^{a}=-g\left(\partial G / \partial \phi_{k}\right)\left(\tau^{a}\right)_{k}^{l} \phi_{l}$ and $g$ is the gauge coupling constant corresponding to each gauge group and $\tau^{a}$ are corresponding generators. For $S U(2)_{L}$ symmetry $\tau^{a}=\sigma^{a} / 2$, where $\sigma^{a}$ are Pauli matrices. And the $U(1)_{Y}$ hypercharges of the fields $H_{u}, H_{d}, L, Q, \tilde{e}_{R}, \tilde{d}_{R}$, and $\tilde{u}_{R}$ given in (3) are $Y=(1 / 2,-1 / 2,-1 / 2,1 / 6,1,1 / 3,-2 / 3)$, respectively. The quantity $f_{a b}$ is related to the kinetic energy of the gauge fields and is a holomorphic function of superfields $\phi_{i}$. The kinetic term of the scalar fields is given by

$$
\mathscr{L}_{\mathrm{KE}}=K_{i}^{j *} \partial_{\mu} \phi^{i} \partial^{\mu} \phi_{j}^{*}
$$

where $K_{j *}^{i}$ is the inverse of the Kähler metric $K_{i}^{j *} \equiv \partial^{2} K / \partial \phi^{i}$ $\partial \phi_{j}^{*}$. We assume that during inflation, SUSY is unbroken and the hidden sector field is subdominant compared to inflaton field so that we can safely assume $Z=0$. Also, for charge conservation, we assume that during inflation the charged fields take zero vev. The $D$-term and $F$-term potentials are obtained as

$$
\begin{aligned}
& V_{D}=\frac{9}{8}\left(g_{1}^{2}+g_{2}^{2}\right) \frac{\left(\left|\phi_{d}\right|^{2}-\left|\phi_{u}\right|^{2}+\left|\phi_{v}\right|^{2}\right)^{2}\left(-1+\zeta\left|\phi_{d}\right|^{2}\right)^{2}}{\left(-3+\left|\phi_{d}\right|^{2}+\left|\phi_{u}\right|^{2}+\left|\phi_{v}\right|^{2}\right)^{2}} \\
& V_{F}=\frac{3 \mu^{2}\left(3\left|\phi_{u}\right|^{2}+\left|\phi_{d}\right|^{2}\left(3-\left|\phi_{u}\right|^{2}\right)\right)}{\left(-3+\left|\phi_{d}\right|^{2}+\left|\phi_{u}\right|^{2}+\left|\phi_{v}\right|^{2}\right)^{2}}
\end{aligned}
$$

It can be seen that the above expressions reduce to MSSM $D$-term and $F$-term potentials in the small field limit after the end of inflation as the terms coming from Kähler potential and gauge kinetic function are Planck suppressed. Now, for simplification, we parametrize the neutral component fields as

$$
\phi_{u}^{0}=\phi \sin [\beta], \phi_{d}^{0}=\phi \cos [\beta], \phi_{v}=\gamma \phi
$$

For the above parametrization, the kinetic term turns out to be

$$
\mathscr{L}_{\mathrm{KE}}=\frac{9\left(1+\gamma^{2}\right)}{\left(-3+\left(1+\gamma^{2}\right)|\phi|^{2}\right)^{2}}\left|\partial_{\mu} \phi\right|^{2} .
$$

To obtain the canonical kinetic term for the inflaton field and to better understand the inflation potential, we redefine the field $\phi$ to $\chi_{c}$ via

$$
\phi=\frac{\sqrt{3}}{\sqrt{1+\gamma^{2}}} \tanh \left(\frac{\chi_{c}}{\sqrt{3}}\right) .
$$

For the above field redefinition, the kinetic term becomes

$$
\mathscr{L}_{\mathrm{KE}}=\sec h^{2}\left(\frac{2 \operatorname{Im}\left[\chi_{c}\right]}{\sqrt{3}}\right)\left|\partial_{\mu} \chi_{c}\right|^{2} .
$$

Therefore, if the imaginary part of the field $\chi_{c}$ is zero, we obtain the canonical kinetic term in real part of field $\chi_{c}=\chi$ (say). As the field $\phi$ is a linear combination of the Higgs and sneutrino field, so is the inflaton field $\chi$, we can call this model a Higgs-sneutrino inflation model. The $D$-term potential (9) in the canonical inflaton field $\chi$ becomes 


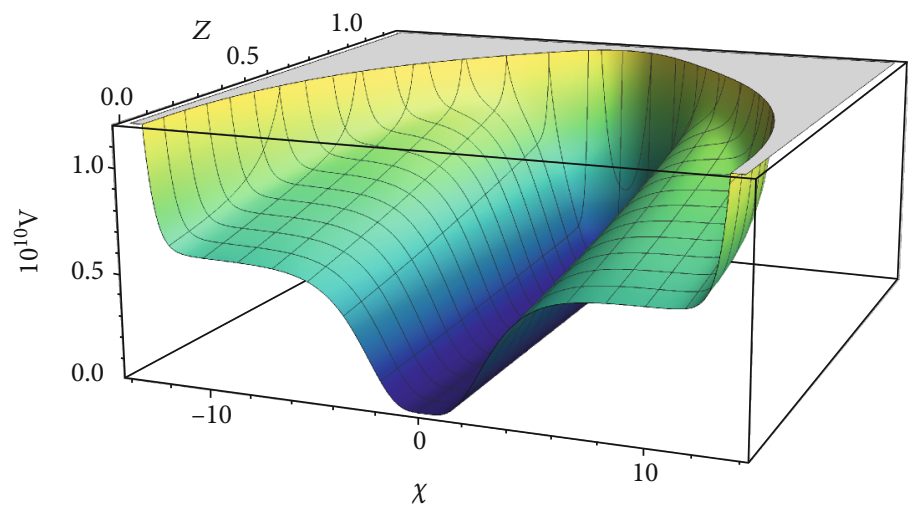

FIGURE 1: The $D$-term inflation potential for $\tan (\beta)=1.8, g_{1}=g_{2}=0.62$ is shown. During inflation, as the inflaton rolls down from $\chi_{i} \simeq 7$ to $\chi_{e} \simeq 1.9$, the potential along the $Z$ direction stays nearly flat implying inflaton $\chi$ being much heavier than the Polonyi field $Z$. For $Z \gtrsim 1$, the potential becomes very steep where slow-roll inflation cannot be achieved.

$$
V_{D}=\frac{1}{2} \lambda^{2}\left(g_{1}^{2}+g_{2}^{2}\right) \tanh ^{4}\left(\frac{\chi}{\sqrt{6}}\right)
$$

where $\lambda=(3 / 2)\left(\left(\gamma^{2}+\cos (2 \beta)\right) /\left(1+\gamma^{2}\right)\right)$ and we have made a specific choice $\zeta=\left(\gamma^{2}+1\right) /(3 \sin (\beta) \cos (\beta))$ which is critical to obtain a plateau behavior potential at large field values which can fix inflationary observables. Since at the GUT scale, the mass parameter $\mu \sim 0$ and $S U(2)$ gauge couplings are $g_{1}=g_{2}=0.62$, the $D$-term potential dominates over $F$ -term potential during inflation when the field values are at the Planck scale. With the canonical kinetic term and scalar potential obtained in canonical inflaton field $\chi$, the theory is now in the Einstein frame. Therefore, we can use the standard Einstein frame relations to estimate the inflationary observables, namely, amplitude of the curvature perturbation $\Delta_{\mathscr{R}}^{2}$, scalar spectral index $n_{s}$ and its running $\alpha_{s}$, and tensor-toscalar ratio $r$, given by

$$
\begin{aligned}
\Delta_{\mathscr{R}}^{2} & =\frac{1}{24 \pi^{2}} \frac{V_{D}}{\varepsilon}, \\
n_{s} & =1-6 \varepsilon+2 \eta, \\
\alpha_{s} & \equiv \frac{d n_{s}}{d \ln k}=16 \varepsilon \eta-24 \varepsilon^{2}-2 \xi, \\
r & =16 \varepsilon,
\end{aligned}
$$

respectively. Here, $\varepsilon, \eta$, and $\xi$ are the slow-roll parameters, given by

$$
\begin{aligned}
& \varepsilon=\frac{1}{2}\left(\frac{V_{D}{ }^{\prime}}{V_{D}}\right)^{2}, \\
& \eta=\frac{V_{D}{ }^{\prime \prime}}{V_{D}}, \\
& \xi=\frac{V_{D}{ }^{\prime} V_{D}{ }^{\prime \prime}}{V_{D}^{2}} .
\end{aligned}
$$

In order to have flat universe as observed, the universe must expand at least by more than 60 -folds during infla- tion. The displacement in the inflaton field during inflation is $\Delta \chi \equiv \chi_{i}-\chi_{f}$.

The field value $\chi_{i}$ is at the onset of inflation, when observable $\mathrm{CMB}$ modes start leaving the horizon, which can be determined using the following relation

$$
N=\int_{\chi_{f}}^{\chi_{i}} \frac{V_{D}}{V_{D}{ }^{\prime}} d \chi
$$

once we fix the field value $\chi_{f}$ using the condition $\varepsilon\left(\chi_{f}\right)=1$ which corresponds to the end of inflation.

From the Planck 2018 CMB temperature anisotropy data in combination with the $\mathrm{EE}$ measurement at low multipoles, we have the scalar amplitude, the spectral index, and its running as $\ln \left(10^{10} \Delta_{\mathscr{R}}^{2}\right)=3.044 \pm 0.014, n_{s}=0.9649 \pm 0.0042$, and $\alpha_{s}=-0.0045 \pm 0.0067$, respectively (68\% CL, PlanckTT,TE,EE+lowE+lensing) [59-61]. Also, the Planck 2018 data combined with BK15 CMB polarization data put an upper bound on tensor-to-scalar ratio $r_{0.002}<0.056$ (95\% CL, PlanckTT,TE,EE+lowE+lensing+BK15) [62, 63]. Armed with the theoretical and observational results, we perform the numerical analysis of the models. From the condition of the end of inflation $\varepsilon=1$, we find $\chi_{e} \simeq 1.927$. Therefore, requiring $N \simeq 60 e$-fold expansion during inflation, we obtain $\chi_{i} \simeq 7.03$. At $\chi_{i} \simeq 7.03, \mathrm{CMB}$ observables are estimated to be $r \simeq 0.0035, n_{s} \simeq 0.965$, and $\alpha_{s} \simeq-6 \times 10^{-4}$, consistent with the observations. The $\left(n_{s}, r\right)$ predictions are similar to the Starobinsky inflation. For $\tan (\beta)=1.8$ and $g_{1}=g_{2}=0.62$, the observed CMB amplitude can be obtained for $\lambda \simeq 1.703$ $\times 10^{-5}$. From the specific case considered in (15) for successful inflation, we have sneutrino field parameter $\gamma \simeq 0.727$ and coupling $\zeta$ of the Planck suppressed operator in the guage kinetic function (2) $\zeta \simeq 1.2$. The evolution of the $D$-term inflaton potential (15) is shown in Figure 1. During inflation, as the inflaton rolls down from $\chi_{i} \simeq 7$ to $\chi_{e} \simeq 1.9$, the potential along the $Z$ direction stays nearly flat implying $\chi$ being much heavier than $Z$. During slow-roll phase, the mass of inflaton varies from $m_{\chi_{i}} \simeq 2.1 \times 10^{13} \mathrm{GeV}$ to $m_{\chi_{e}} \simeq 9.3 \times 1$ $0^{12} \mathrm{GeV}$, whereas the mass parameter associated with the Polonyi field $Z$ varies from $m_{Z_{i}} \simeq 6.1 \times 10^{10} \mathrm{GeV}$ to $m_{Z_{e}} \simeq$ 
$2.1 \times 10^{8} \mathrm{GeV}$. Hence, $m_{Z}^{2} \ll m_{\chi}^{2}$, our assumption that $Z$ is subdominant during inflation and contributes insignificantly to the supergravity inflation potential.

In the next section, we will see that the value of $\tan (\beta)$ $=1.8$ used to fix $\mathrm{CMB}$ amplitude is absolutely critical in order to get PeV dark matter whose decay explains PeV neutrino events observed at IceCube and reproducing lowenergy particle mass spectrum as shown in Table 1. It is important to mention here that the scalar field parameter values $\tan (\beta)=1.8$ and $\gamma \simeq 0.727$ are not a unique set of value to obtain the observed CMB amplitude. Instead, it can be obtained for all the pair of values of $(\gamma, \beta)$ which satisfy the relation $(3 / 2)\left(\left(\gamma^{2}+\cos (2 \beta)\right) /\left(1+\gamma^{2}\right)\right)=\lambda \simeq 1.703$ $\times 10^{-5}$ defined in (15). However, $\tan (\beta)=1.8$ fixed from the requirement of $\mathrm{PeV}$ scale SUSY breaking removes this degeneracy in $(\gamma, \beta)$ in obtaining the correct CMB amplitude, and at the same time, it brings the successful explanation for inflation, dark matter relic density, and $\mathrm{PeV}$ neutrino events at IceCube within the same framework.

\section{SUSY Breaking}

We assumed that during inflation all the fields including the hidden sector field $Z$ are subdominant compared to inflaton field and supersymmetry is unbroken at the time of inflation. Once the inflation ends, the scalar fields effectively become vanishing and the soft mass terms are generated via SUSY breaking as the field $Z$ settles down to a finite minimum of the Polonyi potential. The Polonyi field $Z$ is associated with the fluctuations in the overall size of the compactified dimensions. For the successful implementation of inflation in supergravity, the strong stabilization of the Polonyi field is required which can be achieved via appropriate choice of Kähler potential, gauge kinetic function, and superpotential in $Z$. This also allows for a solution of the cosmological Polonyi problem $[114,115]$ (which is a special case of cosmological moduli problem $[116,117])$ associated with the problem of cosmological nucleosynthesis. Technically, cosmological Polonyi problem is evaded if $Z$ is heavy $m_{Z} \sim \mathcal{O}(100-1000)$ $\mathrm{TeV}$ and the Polonyi mass is much larger than the gravitino mass, i.e., $m_{Z}^{2} \gg m_{3 / 2}^{2}[70,118,119]$. We will see that this problem does not occur in this model.

The appropriately chosen potentials $K\left(Z, Z^{*}\right)$ and $W(Z)$ are shown in equations (1) and (3) and the gauge kinetic function in equation (2). The kinetic term for Polonyi field $Z$ is obtained as $\left(1+2 \alpha|Z|^{2}\right)\left|\partial_{\mu} Z\right|^{2}$ and the Polonyi potential is obtained as

$$
\begin{aligned}
V\left(Z, Z^{*}\right)= & e^{|Z|^{2}+(\alpha / 2)|Z|^{4}}|Z|^{2}\left|\mu_{z z} Z^{2}+\mu_{z}^{2}\right|^{2}\left[-3+\frac{1}{1+2 \alpha|Z|^{2}} \mid \frac{1}{Z}\right. \\
& \left.+Z^{*}+\alpha Z Z^{* 2}+\left.\frac{2 \mu_{z z} Z}{\mu_{z z} Z^{2}+\mu_{z}^{2}}\right|^{2}\right] .
\end{aligned}
$$

We take the hidden sector Polonyi field $Z$ to be real. The parameters $\alpha, \mu_{z}$, and $\mu_{z z}$ are fixed from the requirement that $Z$ which breaks supersymmetry acquire a minima where the
TABLE 1: Particle mass spectrum after EWSB for the benchmark point given in Table 2.

\begin{tabular}{lc}
\hline SUSY fields & Masses in PeV \\
\hline$\tilde{d}_{i L, R}(i=d, s, b)$ & $M_{\tilde{d}_{i, L, R}} \approx(15.75,16.78)_{d}(16.78,16.78)_{s}(17.38,17.38)_{b}$ \\
$\tilde{u}_{i L, R}(i=u, c, t)$ & $M_{\tilde{u}_{i L, R}} \approx(13.30,15.75)_{u}(16.88,16.88)_{c}(17.38,17.38)_{t}$ \\
$\tilde{e}_{i L, R}(i=e, \mu, \tau)$ & $M_{\tilde{e}_{i L, R}} \approx(14.36,14.36)_{e}(14.36,14.86)_{\mu}(14.86,14.86)_{\tau}$ \\
$\tilde{v}_{i}(i=e, \mu, \tau)$ & $M_{\tilde{v}_{i}} \approx(14.86)_{e}(14.86)_{\mu}(14.86)_{\tau}$ \\
$h$ & $M_{h} \approx 126(\mathrm{GeV})$ \\
$H$ & $M_{H} \approx 19.48$ \\
$A$ & $M_{A} \approx 19.48$ \\
$H^{-}$ & $M_{H^{-}} \approx 19.47$ \\
$\tilde{g}_{i}(i=1 \ldots 8)$ & $M_{\tilde{g}_{i}} \approx 14.28$ \\
& $M_{\tilde{\chi}_{1}}(D M) \approx 6.3$ \\
$\tilde{\chi}^{0}(i=1 \ldots 4)$ & $M_{\tilde{\chi}_{2}} \approx 7.22$ \\
& $M_{\tilde{\chi}_{3}} \approx 7.22$ \\
& $M_{\tilde{\chi}_{4}} \approx 9.22$ \\
$\tilde{\chi}^{-}$ & $M_{\tilde{\chi}_{1}^{-}} \approx 7.22$ \\
\hline & $M_{\tilde{\chi}_{2}^{-}} \approx 9.21$
\end{tabular}

gravitino mass is $\sim \mathcal{O}(\mathrm{PeV})$ scale and the Polony potential is positive $V_{F}(Z) \| 0$ with a field minimum at $Z=Z_{\text {min }}$. We find the gravitino mass to be

$$
m_{3 / 2}^{2}=e^{G}=e^{Z^{2}+(\alpha / 2) Z^{4}}\left(\mu_{z z} Z^{3}+\mu_{z}^{2} Z\right)^{2}
$$

and the scalar masses, using $m_{\phi}^{2}=\partial_{\phi} \partial_{\phi^{*}\left(V_{F}\left(\phi, \phi^{*}, Z\right)\right)} / \partial_{\phi} \partial_{\phi^{*}} K$ evaluated at $\phi=0, Z=Z_{\min }$, are obtained as

$$
\begin{aligned}
m_{\phi_{i}}^{2} & =m_{3 / 2}^{2}\left[-2+\frac{1}{1+2 \alpha Z^{2}}\left(\frac{1}{Z}+Z+\alpha Z^{3}+\frac{2 \mu_{z z} Z}{\mu_{z z} Z^{2}+\mu_{z}^{2}}\right)^{2}\right], \\
m_{\phi_{(u, d)}}^{2} & =m_{\phi_{i}}^{2}+\frac{\mu^{2}}{\mu_{z z} Z^{3}+\mu_{Z}^{2} Z^{2}} m_{3 / 2}^{2},
\end{aligned}
$$

where $\phi_{i}=\phi_{v}, \phi_{e}, \phi_{u}^{+}, \phi_{d}^{-}, \tilde{u}_{L}, \tilde{d}_{L}, \tilde{u}_{R}, \tilde{d}_{R}, \tilde{e}_{R}$. At the GUT energy scale, $\mu \sim 0$, all the scalar masses are equal $m_{\phi_{(u, d)}}^{2}=m_{\phi_{i}}^{2}$.

Now, we calculate the coefficients of the soft SUSY breaking terms which arise from the Kähler potential (1) and superpotential (3). The effective potential of the observable scalar sector consists of soft mass terms which give scalar masses as given by equations (24) and (25) and trilinear and bilinear soft SUSY breaking terms given by $[120-122]$

$$
\frac{1}{3} A_{i j k} \phi^{i} \phi^{j} \phi^{k}+\frac{1}{2} B_{i j} \phi^{i} \phi^{j}+\text { h.c. }
$$




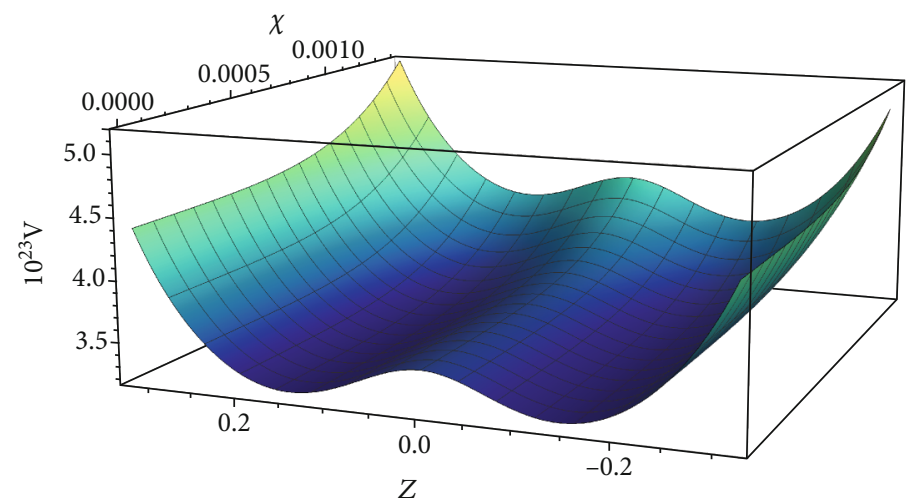

FIgURe 2: After the end of inflation, as $\chi$ approaches minimum near zero, Polonyi field $Z$ settles down to minimum at $Z_{\min } \simeq \pm 0.144$. The positive Polonyi potential is obtained for the parameter values $\alpha=20, \mu_{z} \simeq 2.43 \times 10^{-6}, \mu_{z z} \simeq 2.858 \times 10^{-11}$, and $\kappa=0.56$, which are fixed from the requirement of getting PeV scale soft SUSY breaking parameters.

Here, the coefficients $A_{i j k}$ and $B_{i j}$ are given by

$$
\begin{aligned}
A_{i j k} & =\left[\frac{\partial_{Z^{*}} W \wedge^{*}+W \wedge^{*} \partial_{Z^{*}} \widehat{K}}{\partial_{Z^{*}} \partial_{Z} \widehat{K}} \partial_{Z} e^{K \wedge}\right] \tilde{Y}_{i j k}, \\
B_{i j} & =\left[\frac{\partial_{Z^{*}} W \wedge^{*}+W \wedge^{*} \partial_{Z^{*}} \widehat{K}}{\partial_{Z^{*}} \partial_{Z} \widehat{K}} \partial_{Z} e^{K \wedge}-m_{\frac{3}{2}} e^{K \wedge / 2}\right] \tilde{\mu}_{i j} .
\end{aligned}
$$

From equations (1) and (3), we have $\widehat{K}\left(Z, Z^{*}\right)=Z Z^{*}$ $+(\alpha / 2)\left(Z Z^{*}\right)^{2}$ and $\widehat{W}(Z)=\mu_{z}^{2} Z+\mu_{z z} Z^{3}$. The coefficients of normalized masses $\tilde{\mu}_{i j}$ and Yukawa couplings $\tilde{Y}_{i j k}$ in the trilinear (26) and bilinear (27) terms, respectively, are obtained as

$$
\begin{aligned}
& A_{0}=e^{Z^{2}+(\alpha / 2) Z^{4}} \frac{Z\left(1+Z^{2} \alpha\right)\left[\mu_{z z} Z^{2}\left(3+Z^{2}+Z^{4} \alpha\right)+\left(1+Z^{2}+Z^{4} \alpha\right) \mu_{z}^{2}\right]}{1+2 \alpha Z^{2}}, \\
& B_{0}=A_{0}-e^{Z^{2}+(\alpha / 2) Z^{4}}\left(\mu_{z z} Z^{3}+\mu_{z}^{2} Z\right) .
\end{aligned}
$$

We drop the $B_{i j}$ contribution to the scalar masses by taking corresponding $\tilde{\mu}_{i j}$ small. Also, from the fermionic part of the SUGRA Lagrangian, the soft gaugino masses can be obtained as [122-124]

$$
m_{1 / 2}=\frac{1}{2}\left[\operatorname{Re} f_{a b}\right]^{-1} e^{G / 2} \partial_{I} f_{a b} K \Lambda^{I J^{*}} G_{J^{*}}
$$

For the choice of gauge kinetic function $f_{a b}=e^{-\kappa Z} \delta_{a b}$ when $\phi \approx 0$ after inflation, we obtain the gaugino mass

$$
m_{1 / 2}=\frac{\kappa}{2} m_{3 / 2}\left(1+2 \alpha Z^{2}\right)\left(Z+\alpha Z^{3}+\frac{\mu_{z}^{2}+3 \mu_{z z} Z^{2}}{\mu_{z}^{2} Z+\mu_{z z} Z^{3}}\right) .
$$

We show that the Polonyi potential (22) in Figure 2 for the parameters $\alpha=20, \mu_{z} \simeq 2.43 \times 10^{-6}, \mu_{z z} \simeq 2.858 \times 1$ $0^{-11}$, and $\kappa=0.56$ has a minimum at $Z_{\min } \simeq \pm 0.144$. These parameter values are fixed at the GUT scale from the requirement to achieve the soft SUSY breaking parameters

$$
\begin{aligned}
A_{0} & =-2.2 \mathrm{PeV}, \\
m_{0} & =m_{\phi}=14 \mathrm{PeV}, \\
m_{1 / 2} & \simeq 10 \mathrm{PeV},
\end{aligned}
$$

with the specific choice of $\tan \beta=1.8$ and sign of $\mu>0$ which gives the Higgs mass $\sim 125 \mathrm{GeV}$ at electroweak scale satisfying all experimental (LHC, etc.) and theoretical constraints (stability, unitarity, etc.). The gravitino mass and the mass of the Polonyi field come out to be $m_{3 / 2} \simeq$ $2.32 \mathrm{PeV}$ and $m_{z} \simeq 31.8 \mathrm{PeV}$, respectively, which implies $m_{Z}^{2} \gg m_{3 / 2}^{2}$ and therefore the $\mathcal{O}(\mathrm{PeV})$ scale oscillations of the Polonyi field near its minimum decay much before the Big Bang nucleosynthesis. This leads to strong stabilization of $Z$, and therefore, the cosmological Polonyi problem does not arise in this model.

Knowing the soft breaking parameters at a highenergy scale does not tell us anything about the phenomenology we could observe in the experiments such at LHC, direct detection of DM, and IceCube. We need to find these parameters at the low energy. In general, all parameters appearing in the supersymmetric Lagrangian evolve with RGEs. These RGEs are the intermediator between the unified theory at GUT scale and the lowenergy masses and couplings, which strictly depend on the boundary conditions. We apply RGEs to calculate low-energy masses and different branching ratios for the abovementioned set of mSUGRA parameters as the RGEs are coupled differential equations, which cannot be solved analytically. Also, the low-energy phenomenology is complicated due to mixing angles and dependence of couplings on the high-scale parameters; therefore, one has to rely on numerical techniques to solve RGEs. We calculate all the variables as allowed by the present experimental data. There are various programs publicly available such as SARAH, SPheno, and Suspect which can generate two-loop RGEs and calculate the mass spectrum and the couplings at low energy. For this work, we use SARAH 


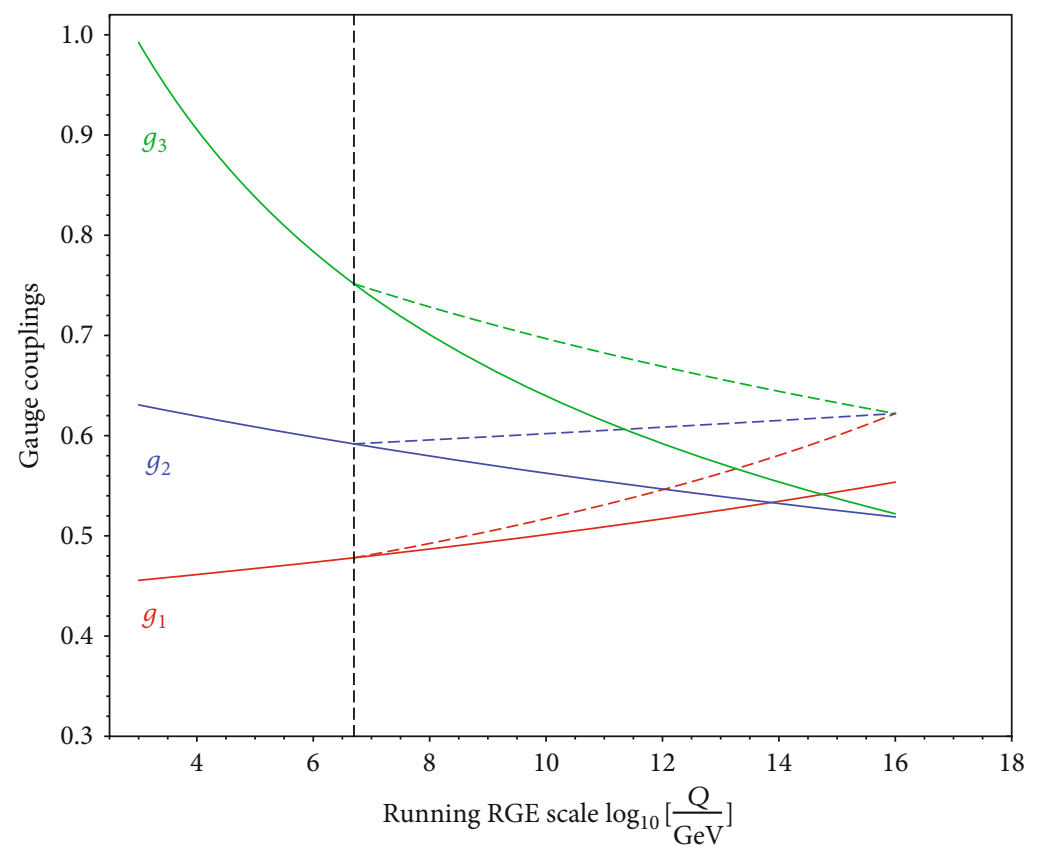

FIGURE 3: Solid lines represent the gauge coupling evolution for the standard model. Dotted lines represent the gauge coupling unification for our benchmark points (Table 2).

4.11.0 $[89,90]$ to generate the RGEs and other input files for SPheno 4.0.2 [91, 92] which generate the mass spectrum, couplings, branching ratios, and decay widths of supersymmetric particles. To study the neutralino as a dark matter candidate, we link the output files of SPheno and model files from SARAH to micrOMEGAs 3.6.8 [93, 94] to calculate the number density for a $\mathrm{PeV}$ neutralino dark matter. In the next section, we discuss the consequences of a $\mathrm{PeV}$ dark matter.

\section{Bino-Dominated DM in the mSUGRA Model}

The neutralinos $\chi_{i}(i=1,2,3,4)$ are the physical superpositions of two gauginos, namely, bino $\tilde{B}$ and wino $\tilde{W}_{3}$, and two higgsinos $\tilde{H}_{u 0}$ and $\tilde{H}_{d 0}$. The neutralino mass matrix is given by

$$
M_{N}=\left(\begin{array}{cccc}
M_{1} & 0 & -M_{Z} c_{b} s_{\theta} & M_{Z} s_{b} s_{\theta} \\
0 & M_{2} & M_{Z} c_{b} c_{\theta} & -M_{Z} s_{b} c_{\theta} \\
-M_{Z} c_{b} s_{\theta} & M_{Z} c_{b} c_{\theta} & 0 & -\mu \\
M_{Z} s_{b} s_{\theta} & -M_{Z} s_{b} c_{\theta} & -\mu & 0
\end{array}\right),
$$

where $c_{b} \equiv \cos \beta, c_{\theta} \equiv \cos \theta_{W}$, and $\theta_{W}$ is the Weinberg mixing angle. $M_{1}$ and $M_{2}$ are the bino and wino mass parameter at the EWSB scale. The lightest eigenvalue of the above matrix and the corresponding mass eigenstate has good chance of being the LSP. The higgsinos dominantly contribute in LSP for $|\mu|<M_{1,2}$, whereas for $|\mu|>$
$M_{1,2}$, the LSP can be determined by bino and wino. The lightest neutralino becomes bino-like when $M_{2}>M_{1}$. In the mSUGRA model, $M_{1}$ and $M_{2}$ are equal due to the universality of the gaugino masses at the GUT scale where the value of the gauge coupling constants $g_{1}, g_{2}$, and $g_{3}$ becomes equal; see Figure 3. However, at low energy, $M_{2} \approx\left(5 g_{2}^{2}\left(M_{Z}\right) / 3 g_{1}^{2}\left(M_{Z}\right)\right) M_{1}$ [110]. This implies that the LSP is mostly bino-like.

4.1. Relic Density and PeV Excess at IceCube. In this analysis, we present our mSUGRA model with the aim to realize a $\mathrm{PeV}$ scale dark matter candidate, which can also explain the IceCube HESE events. IceCube [26-29] recently reported their observation of high-energy neutrinos in the range $30 \mathrm{TeV}-2 \mathrm{PeV}$. The observation of neutrinos is isotropic in arrival directions. No particular pattern has been identified in arrival times. This implies that the source is not local but broadly distributed. This superheavy neutrino (LSP) might be the dark matter, distributed in the Galactic halo. In this work, we find that the thermally produced LSP can serve as a viable PeV dark matter candidate satisfying the dark matter relic density in the right ball park which also explains the IceCube excess at PeV. However, an annihilating PeV scale dark matter candidate possesses two serious problems.

(1) To maintain the correct relic density using the wellknown thermal freeze-out mechanism requires a very large annihilation cross section, which violates the unitarity bound. The $s$-wave annihilation cross section of dark matter with a mass $M_{\mathrm{DM}}$ is limited by unitarity as [125] 


$$
\langle\sigma v\rangle \leq \frac{4 \pi}{M_{\mathrm{DM}}^{2} v}
$$

(i) The unitarity bound restrict the dark matter mass below $300 \mathrm{TeV}$ [125]. Also, in order to satisfy the unitarity bound, the PeV scale dark matter model produces an overabundance $\mathcal{O}\left(10^{8}\right)$ (depends on the model) of the dark matter

(2) The decay time of a particle is in general inversely proportional to its mass. So a $\mathrm{PeV}$ scale particle is generically too short-lived to be a dark matter candidate. One has to use fine-tuning $[126,127]$ to stabilize the dark matter as lifetime of the DM particles has to be at least larger than the age of the universe $[29,128]$

Generally, thermal dark matter freeze-out depends on the remaining dark matter in chemical and thermal equilibrium with the SM bath, which leads to depletion of dark matter through Boltzmann suppression [129, 130]. We consider the possibility that the dark matter can also decay out of equilibrium to the SM particles via $R$-parity violation. In the presence of constant $s$-wave effective annihilation cross section and dark matter decay, the Boltzmann equation is given by

$$
\frac{d n}{d t}=-3 H n-\langle\sigma v\rangle\left(n^{2}-n_{\mathrm{eq}}^{2}\right)-n \sum_{i} \frac{1}{\tau_{i, \mathrm{DM}}} .
$$

Here, we assume that the decay rates of super partner of SM particles other than LSP are much faster than the rate of the expansion of the universe, so that all the particles present at the beginning of the universe have decayed into the lightest neutralino before the freeze-out. Therefore, the density of the lightest neutralino $n$ is the sum of the density of all SUSY particles. $\tau_{i, \mathrm{DM}}$ is the decay time for the $i^{\text {th }}$ process. In nonrelativistic case $T<M_{\mathrm{DM}}$, the equilibrium number density $n_{\mathrm{eq}}$ is given by the classical Maxwell-Boltzmann distribution:

$$
n_{\mathrm{eq}}=\left(\frac{M_{\mathrm{DM}} T}{2 \pi}\right)^{3 / 2} \operatorname{Exp}\left(-\frac{M_{\mathrm{DM}}}{T}\right) .
$$

The entropy density of the universe at temperature $T$ is $s=g_{*} T^{3}\left(2 \pi^{2} / 45\right)$, where parameter $g_{*}$ is the effective degrees of freedom. We use the relation of entropy density and the expansion rate of the universe $(d s / d t)+3 H s=0$, in equation (28), to obtain

$$
\frac{d Y}{d t}=s\langle\sigma v\rangle\left(Y^{2}-Y_{\mathrm{eq}}^{2}\right)-Y \sum_{i} \frac{1}{\tau_{i, \mathrm{DM}}},
$$

where yield $Y$ is defined as $Y \equiv n / s$. We solve the above equation for the decaying LSP with large decay time. Before freeze-out annihilation term in equation (30) dominates over the exponential decay term due to very large decay time of LSP. Therefore, integrating equation (30) between the times $t=0$ (or $T=\infty$ ) the start of the universe and $t=t_{f}$ (or $T=T_{f}$ ) the freeze-out time, the yield $Y\left(t_{f}\right)$ comes out to be inversely proportional to the thermally averaged effective annihilation cross section $\langle\sigma v\rangle$ of the dark matter [131]. However, after freeze-out, the annihilation of LSP is no longer large enough and decay term becomes dominant. Therefore, we neglect the annihilation term compared to the decay term and integrate the remaining equation between the freeze-out time $t_{f}$ and the present age of the universe $\tau_{U}$, which, for $t_{U}>>t_{f}$, gives the yield today as

$$
Y\left(T_{0}\right)=Y\left(T_{f}\right) \operatorname{Exp}\left(-\sum_{i} \frac{t_{U}}{\tau_{i, \mathrm{DM}}}\right),
$$

which implies that the number density of LSP reduces with time. Therefore, the relic abundance of LSP in the present universe can be written as

$$
\Omega=\frac{s_{0}}{\rho_{c}} M_{\mathrm{DM}} Y\left(T_{0}\right)=\frac{s_{0}}{\rho_{c}} M_{\mathrm{DM}} Y\left(T_{f}\right) \operatorname{Exp}\left(-\sum_{i} \frac{t_{U}}{\tau_{i, \mathrm{DM}}}\right),
$$

where $s_{0} \sim 2890 \mathrm{~cm}^{-3}$ is the current entropy density and $\rho_{c} \sim 1.05 \times 10^{-5} h^{2} \mathrm{GeV} \mathrm{cm}^{-3}$ is the critical density of the present universe, $h=0.72$ is a dimensionless parameter defined through the Hubble parameter $H_{0}=100 \mathrm{~h} \mathrm{~km} \mathrm{se}$ $\mathrm{c}^{-1} \mathrm{Mpc}^{-1}$. This implies that the relic density is related to the annihilation cross section and the decay lifetime as

$$
\Omega h^{2} \simeq \frac{9.62 \times 10^{-28}\left[\mathrm{~cm}^{3} \mathrm{sec}^{-1}\right]}{\langle\sigma v\rangle} \operatorname{Exp}\left(-\sum_{i} \frac{t_{U}}{\tau_{i, \mathrm{DM}}}\right) .
$$

Using particle spectrum (see Table 1) in micrOMEGAs, we get

$$
\langle\sigma v\rangle=6.78 \times 10^{-26} \mathrm{~cm}^{3} \mathrm{sec}^{-1}
$$

The main contribution to the DM effective annihilation cross section comes from the coannihilation channel (see Figure 4) $\chi_{1} \chi_{1}^{ \pm} \longrightarrow Z W^{ \pm}$which is consistent with unitarity bound [125] and gives the bino-type DM density $\Omega h^{2} \simeq 0.0142 \operatorname{Exp}\left(-\sum_{i}\left(t_{U} / \tau_{i, \mathrm{DM}}\right)\right)$. The joint results of CMB observations from WMAP and Planck collaboration [132] give $\Omega h^{2}=0.1198 \pm 0.0026$. It has been shown that if a subdominant fraction of DM is decaying [36, 133], then it can resolve the conflict between $\sigma_{8}$ and $H_{0}$ which exists in the $\Lambda \mathrm{CDM}$ model. Berezhiani et al. have shown that $\sim 5 \%$ of the dark matter which has a decay lifetime of $1.6 \times 10^{16}$ seconds resolves the $\sigma_{8}-H_{0}$ conflict [133]. For the PeV DM, Anchordoqui et al. have shown that 

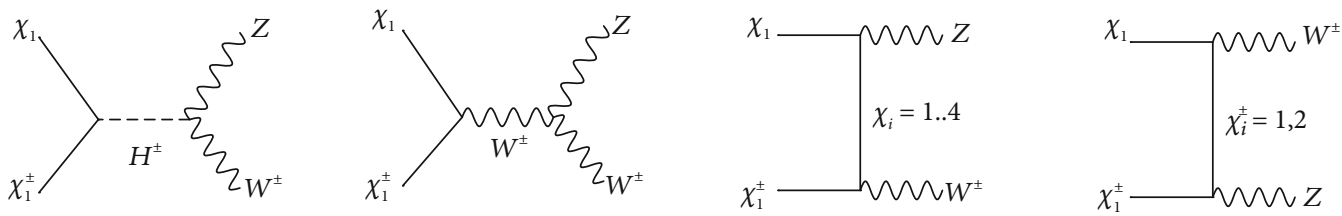

FIGURE 4: Coannihilation diagram of the lightest neutralino $\chi_{1}(\mathrm{DM})$ with the charginos $\chi_{1}^{ \pm}$.
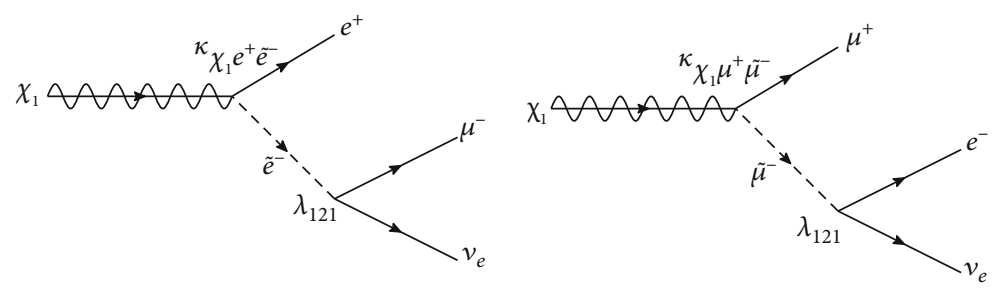

Figure 5: Decay diagrams of neutralino via lepton number violating coupling which produces the IceCube flux.

TABLE 2: A list of the benchmark points (BP) as used in our analysis. Using these BPs, we have reproduced the Higgs mass at $\sim 125 \mathrm{GeV}$ and explain the relic density and the $\mathrm{PeV}$ neutrino events seen at the IceCube experiment.

\begin{tabular}{lcccccc}
\hline \multicolumn{3}{l}{$\begin{array}{l}\text { SUGRA parameters } \\
\text { Masses in PeV }\end{array}$} & & RPV couplings & DM mass in PeV & Decay time in (secs) explains IceCube HESE events \\
$m_{0}$ & $m_{1 / 2}$ & $A_{0}$ & $\tan \beta$ & $\lambda_{121}$ & 6.3 & $8.90 \times 10^{28}$ \\
\hline 14 & 10 & -2.2 & 1.8 & $2.82 \times 10^{-28}$ & 6.3 \\
\hline
\end{tabular}

$\sim 5 \%$ of $\mathrm{DM}$ of mass scale $76 \mathrm{PeV}$ and lifetime $6 \times 10^{15} \mathrm{sec}-$ onds is required for explaining the IceCube events [36]. In this paper, we assume that the lifetime of DM decay to leptons via the $R$-parity violation $L L e^{c}$ operator is $8.9 \times 1$ $0^{28}$ seconds which gives $11 \%$ (i.e., $\Omega h^{2} \simeq 0.0142$ ) of the $\mathrm{DM}$ relic density and is consistent with the structure formation. The neutrino flux is obtained by this decay channel to give the $\mathrm{PeV}$ neutrino flux required to explain the IceCube HESE data [26-29]. We will explain this result in Section 4.2 in more detail.

The presence of $R$-parity violating coupling 5 can explain the high-energy neutrino events at IceCube. We take only one nonzero dimensionless trilinear $R$-parity violating couplings, namely, $\lambda_{121}$ coefficient of $L_{e} L_{\mu} e^{c} . \lambda_{121}$ helps in producing the neutrino flux through the decay of the neutralino (see Figure 5). We find the decay width for the dark matter decaying into $e^{+} \mu^{-} \nu_{e}$ and $e^{-} \mu^{+} \nu_{e}$ [134] as

$$
\begin{aligned}
\Gamma\left(\chi_{1} \longrightarrow e^{+} \mu^{-} \nu_{e}\right) & =\frac{\kappa_{\chi_{1} e^{+} \tilde{e}^{-}}^{2} \lambda_{121}^{2} M_{\chi_{1}}^{5}}{(8 \pi)^{3} M_{\tilde{e}^{-}}^{4}}, \\
\Gamma\left(\chi_{1} \longrightarrow e^{-} \mu^{+} v_{e}\right) & =\frac{\kappa_{\chi_{1} \mu^{+} \tilde{\mu}^{-}}^{2} \lambda_{121}^{2} M_{\chi_{1}}^{5}}{8 \pi^{3} M_{\tilde{\mu}^{-}}^{4}},
\end{aligned}
$$

where coupling $\kappa_{\chi_{1} e^{+} \tilde{e}^{-}}=\kappa_{\chi_{1} \mu^{+} \tilde{\mu}^{-}} \sim \sqrt{2} g_{2}[4,5], M_{\chi_{1}} \simeq 6.3$ $\mathrm{PeV}$, and $M_{\tilde{e}^{-}, \tilde{\mu}^{-}} \simeq 14.36 \mathrm{PeV}$ (see Table 1). For the lepton number violating coupling $\lambda_{121} \approx 2.82 \times 10^{-28}$, we get the decay time for this channel $\tau_{v, \mathrm{DM}} \approx 8.9 \times 10^{28} \mathrm{sec}$ (see Table 2). It is to be noted that we need very tiny $\lambda$ 's to explain the IceCube HESE data. It indeed has the fine-tuning issue, and the dynamical explanation lies somewhere else. To explain the IceCube HESE data in terms of decaying dark matter, fine-tuning actually seems to be the most "natural" option [56, 95-97].

4.2. Fitting the IceCube Data. In this subsection, we fit the flux of neutrinos observed at IceCube from the decay of the neutralino LSP. The total contribution to the neutrino flux from the atmospheric background and the astrophysical sources along with the galactic (G) DM halo and extragalactic (EG) DM is given by

$$
\frac{d \Phi_{\mathrm{tot}}}{d E_{v}}=\frac{d \Phi_{\mathrm{Atm}}}{d E_{v}}+\frac{d \Phi_{\mathrm{astro}}}{d E_{v}}+\frac{d \Phi_{\mathrm{G}}}{d E_{v}}+\frac{d \Phi_{\mathrm{EG}}}{d E_{v}} .
$$

Following the analysis as in Ref. [39] for our mSUGRA model parameters, we compute the number of neutrino events as a function of deposited energy

$$
N_{\text {bin }}=T_{E} \int_{E_{\min }^{\mathrm{bin}}\left(E_{\mathrm{dep}}\right)}^{E_{\max }^{\mathrm{bin}}\left(E_{\mathrm{dep}}\right)} A\left(E_{v}\right) \frac{d \Phi_{\text {tot }}}{d E_{v}} d E_{\mathrm{dep}}\left(E_{v}\right) \text {, }
$$

where $T_{E}=2635$ is the total exposure time, $E_{\mathrm{dep}}$ is the deposited energy in the laboratory frame, and $A\left(E_{v}\right)$ is the neutrino effective area for particular lepton flavor [135]. We sum over all the neutrino flavors. Due to low statistical data points at IceCube, it is acceptable to assume that the two energies coincide, i.e., $E_{\mathrm{dep}} \simeq E_{v}[41]$. 


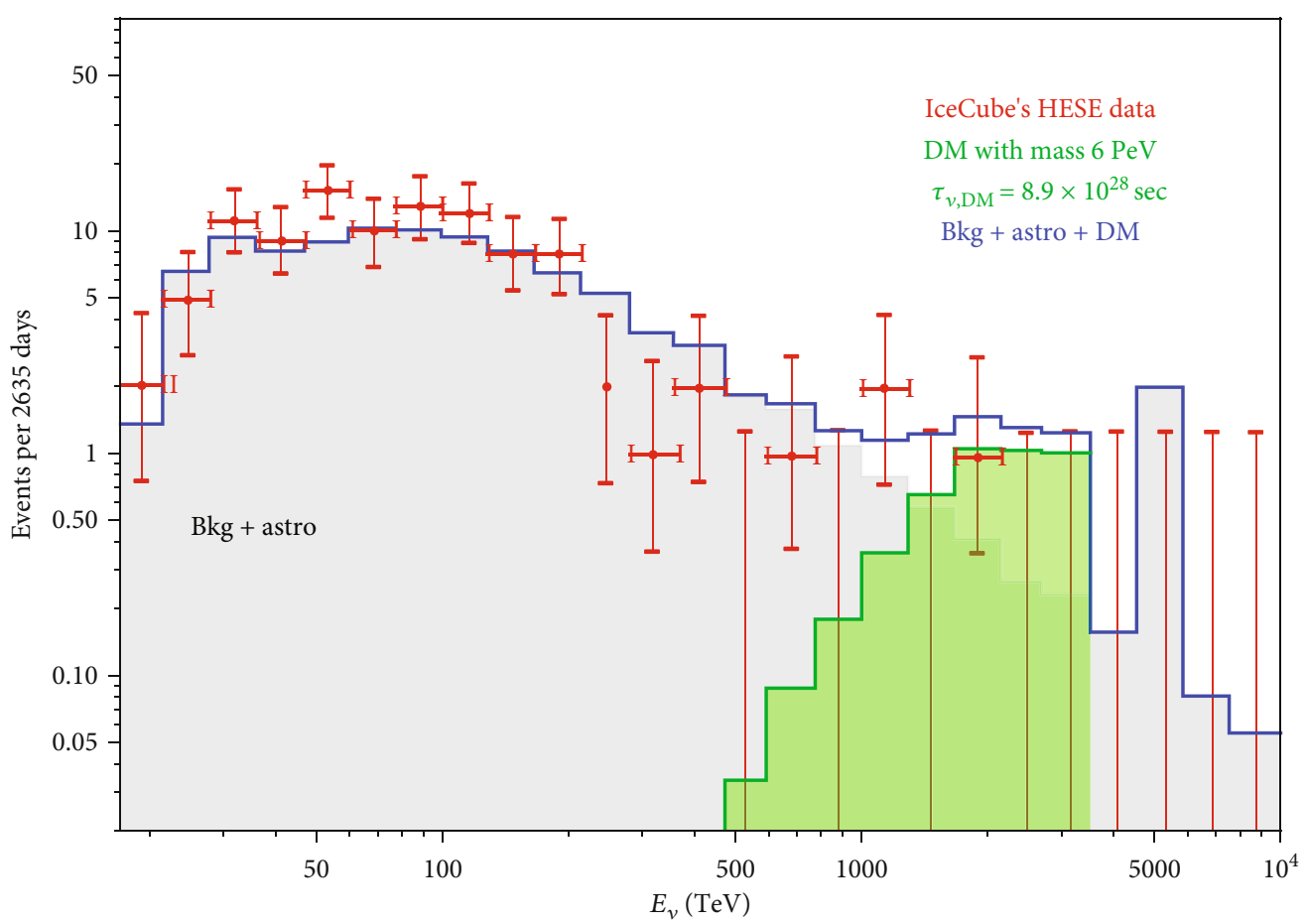

Figure 6: Normalized neutrino event distribution is shown. The contributions to PeV neutrino flux from the galactic and the extragalactic DM decay are shown by the green region. The total flux from all the sources is shown by the blue line.

We have extracted the atmospheric background data from Ref. [136] and added it to the data from astrophysical sources which come mainly due to the decay of highly energetic pions. The source of these pions could be known sources like active galactic nuclei or the Supernova remnants. We use the power law flux $E_{v}^{2}\left(d \Phi_{\text {astro }} / d E_{v}\right)=\Phi_{0}\left(E_{v}\right)^{-\gamma}$ with $\Phi_{0}=2.2 \times 10^{-8} \mathrm{~cm}^{-2} \mathrm{sec}^{-1} \mathrm{sr}^{-1}$ and $\gamma=0.8$ [41] to fit the data from the astrophysical sources. Both the galactic and extragalactic neutrino flux contributions are important to explain the excess neutrino flux around 1-2 PeV [39-43, 53, 135]. We take NFW (Navarro, Frenk, and White) DM density profile to account for the galactic dark matter contribution, where the neutrino energy spectrum $d N / d E_{v}$ evaluated at our model parameter values has been used [137]. We present the neutrino event distribution in Figure 6. The contribution to the neutrino flux from the DM decay can explain the $\mathrm{PeV}$ excess at IceCube.

\section{Conclusions}

In this paper, we have constructed a Starobinsky-like Higgssneutrino model of plateau inflation from supergravity $D$ -term in MSSM fields, and $6 \mathrm{PeV}$ bino-type dark matter gives the observed flux of PeV neutrino events at IceCube HESE. A subdominant fraction $(\sim 11 \%)$ of the DM relic density and its decay to neutrinos is obtained by choosing the couplings of the $R$-parity violating operators. The SUSY breaking is obtained by the Polonyi field which sets the scale of soft SUSY parameters $m_{0}, m_{1 / 2}, A_{0}$, and $m_{3 / 2}$ as a function of the parameters of the model. By running the RGEs, we show the low energy (PeV scale) spectrum of SUSY particles and show that the model can accommodate the $\sim 125 \mathrm{GeV}$ Higgs.
The SUSY spectrum so obtained modifies the RGEs above the PeV scale and gives the coupling constant unification at the GUT scale; however, such a gauge coupling unification can be achieved for a large range of SUSY breaking scales. We find that there is degeneracy in the values of inflation model parameters $(\gamma, \beta, \zeta)$ in predicting the correct $\mathrm{CMB}$ amplitude. However, $\tan (\beta)=1.8$, fixed from SUSY breaking, removes this degeneracy and provides a unique solution for inflation and brings the explanation for dark matter, $\mathrm{PeV}$ neutrinos, and inflation within the same model setup.

\section{Data Availability}

The data used to support the findings of this study are available from the corresponding author upon request.

\section{Conflicts of Interest}

The authors declare that there are no conflicts of interest regarding the publication of this paper.

\section{Acknowledgments}

G.K.C. would like to thank University Grants Commission, Govt. of India, for providing financial support via Dr. D. S. Kothari Postdoctoral Fellowship (Grant No. BSR/PH/201718/0026). N.K. acknowledges private communications with Florian Staub. 


\section{References}

[1] ATLAS collaboration, "Observation of a new particle in the search for the Standard Model Higgs boson with the ATLAS detector at the LHC," Physics Letters B, vol. 716, pp. 1-29, 2012.

[2] P. P. Giardino, K. Kannike, I. Masina, M. Raidal, and A. Strumia, "The universal Higgs fit," Journal of High Energy Physics, vol. 5, p. 46, 2014.

[3] CMS collaboration, "Observation of a new boson at a mass of $125 \mathrm{GeV}$ with the CMS experiment at the LHC," Physics Letters B, vol. 716, pp. 30-61, 2012.

[4] M. Drees, R. Godbole, and P. Roy, Theory and Phenomenology of Sparticles: An Account of Four-Dimensional $N=1$ Supersymmetry in High Energy Physics, 2004.

[5] S. P. Martin, "A supersymmetry primer," in Advanced Series on Directions in High Energy Physics, pp. 1-153, Perspectives on Supersymmetry II, 2010.

[6] H. Baer and X. Tata, Weak Scale Supersymmetry: From Superfields to Scattering Events, Cambridge University Press, 2006.

[7] J. R. Ellis, K. Enqvist, D. V. Nanopoulos, and F. Zwirner, "Observables in low-energy superstring models," Modern Physics Letters A, vol. 1, pp. 57-69, 1986.

[8] R. Barbieri and G. F. Giudice, "Upper bounds on supersymmetric particle masses," Nuclear Physics, vol. 306, no. 1, pp. 63-76, 1988.

[9] ATLAS collaboration collaborationAtlas collaborationhttps://twiki.cern.ch/twiki/bin/view/atlaspublic.

[10] CMS collaboration collaborationCMS collaborationhttp:// cms-results.web.cern.ch/cms-results/public-results/ publications/.

[11] M. R. Buckley, D. Feld, S. Macaluso, A. Monteux, and D. Shih, "Cornering natural SUSY at LHC run II and beyond," Journal of High Energy Physics, vol. 2017, no. 8, article 08059, 2017.

[12] J. L. Feng, "Naturalness and the status of supersymmetry," Annual Review of Nuclear and Particle Science, vol. 63, no. 1, pp. 351-382, 2013.

[13] G. F. Giudice and A. Romanino, "Split supersymmetry," Nuclear Physics B, vol. 699, no. 1-2, pp. 65-89, 2004.

[14] XENON collaboration, "First dark matter search results from the XENON1T experiment," Physical Review Letters, vol. 119, article 181301, 2017.

[15] LUX collaboration, "Results from a search for dark matter in the complete LUX exposure," Physical Review Letters, vol. 118, article 021303, 2017.

[16] GAMBIT collaboration, "Global fits of GUT-scale SUSY models with GAMBIT," The European Physical Journal C, vol. 77, no. 12, 2017.

[17] M. Abdughani, L. Wu, and J. M. Yang, "The mixed binohiggsino dark matter in natural SUSY confronted with XENON1T/PandaX and LHC data," http://arxiv.org/abs/ 1705.09164 .

[18] B. Zhu, R. Ding, and T. Li, "Higgs mass and muon g-2 anomaly in MSSM with gauge-gravity hybrid mediation," http://arxiv.org/abs/1610.09840.

[19] M. Peiro and S. Robles, "Low-mass neutralino dark matter in supergravity scenarios: phenomenology and naturalness," Journal of Cosmology and Astroparticle Physics, vol. 1705, p. 10, 2017.
[20] F. Wang, W. Wang, and J. M. Yang, "Reconcile muon g-2 anomaly with LHC data in SUGRA with generalized gravity mediation," Journal of High Energy Physics, vol. 2015, no. 6, p. 79, 2015.

[21] M. Chakraborti, U. Chattopadhyay, and S. Poddar, "How light a higgsino or a wino dark matter can become in a compressed scenario of MSSM," Journal of High Energy Physics, vol. 2017, no. 9, 2017.

[22] W. Abdallah and S. Khalil, "MSSM dark matter in light of Higgs and LUX results," Advances in High Energy Physics, vol. 2016, Article ID 5687463, 10 pages, 2016.

[23] M. Badziak, M. Olechowski, and P. Szczerbiak, "Is welltempered neutralino in MSSM still alive after 2016 LUX results?," Physics Letters B, vol. 770, pp. 226-235, 2017.

[24] J. Cao, Y. He, L. Shang, W. Su, P. Wu, and Y. Zhang, "Strong constraints of LUX-2016 results on the natural NMSSM," Journal of High Energy Physics, vol. 10, p. 136, 2016.

[25] A. Basirnia, S. Macaluso, and D. Shih, "Dark matter and the Higgs in natural SUSY," Journal of High Energy Physics, vol. 2017, no. 3, p. 73, 2017.

[26] A. Schneider, "Characterization of the astrophysical diffuse neutrino flux with IceCube high-energy starting events," PoS (ICRC2019), vol. 1004, 2020http://arxiv.org/abs/1907 .11266 .

[27] IceCube collaboration, "Evidence for high-energy extraterrestrial neutrinos at the IceCube detector," Science, vol. 342, no. 6161, article 1242856, 2013.

[28] IceCube collaboration, "First observation of PeV-energy neutrinos with IceCube," Physical Review Letters, vol. 111, article 021103, 2013.

[29] IceCube collaboration, "Observation of high-energy astrophysical neutrinos in three years of IceCube data," Physical Review Letters, vol. 113, article 101101, 2014.

[30] K. Murase, "On the origin of high-energy cosmic neutrinos," AIP Conference Proceedings, vol. 1666, article 040006, 2015.

[31] B. Feldstein, A. Kusenko, S. Matsumoto, and T. T. Yanagida, "Neutrinos at IceCube from heavy decaying dark matter," Physical Review D, vol. 88, no. 1, article 015004, 2013.

[32] Y. Sui and P. Bhupal Dev, "A combined astrophysical and dark matter interpretation of the IceCube HESE and throughgoing muon events," Journal of Cosmology and Astroparticle Physics, vol. 2018, no. 7, p. 20, 2018.

[33] M. Kachelriess, O. Kalashev, and M. Kuznetsov, "Heavy decaying dark matter and IceCube high energy neutrinos," Physical Review D, vol. 98, no. 8, article 083016, 2018.

[34] A. Bhattacharya, A. Esmaili, S. Palomares-Ruiz, and I. Sarcevic, "Update on decaying and annihilating heavy dark matter with the 6-year IceCube HESE data," Journal of Cosmology and Astroparticle Physics, vol. 2019, no. 5, p. 51, 2019.

[35] M. Pandey, D. Majumdar, and A. Halderhttp://arxiv.org/abs/ 1909.06839.

[36] L. A. Anchordoqui, V. Barger, H. Goldberg et al., "IceCube neutrinos, decaying dark matter, and the Hubble constant," Physical Review D, vol. 92, no. 6, article 061301, 2015.

[37] IceCube collaboration, "Search for dark matter from the Galactic halo with the IceCube neutrino telescope," Physical Review D, vol. 84, article 022004, 2011.

[38] M. Chianese and A. Merle, "A consistent theory of decaying dark matter connecting IceCube to the Sesame Street," Journal of Cosmology and Astroparticle Physics, vol. 2017, no. 4, p. 17, 2017. 
[39] S. B. Roland, B. Shakya, and J. D. Wells, "PeV neutrinos and a $3.5 \mathrm{keV}$ x-ray line from a PeV-scale supersymmetric neutrino sector," Physical Review D, vol. 92, no. 9, article 095018, 2015.

[40] D. Borah, A. Dasgupta, U. K. Dey, S. Patra, and G. Tomar, "Multi-component fermionic dark matter and IceCube $\mathrm{PeV}$ scale neutrinos in left-right model with gauge unification," Journal of High Energy Physics, vol. 2017, no. 9, 2017.

[41] C. El Aisati, M. Gustafsson, and T. Hambye, "New search for monochromatic neutrinos from dark matter decay," Physical Review D, vol. 92, no. 12, article 123515, 2015.

[42] B. Shakya, S. B. Roland, and J. D. Wells, "Hints of a PeV scale supersymmetric neutrino sector: neutrino masses, dark matter, $\mathrm{keV}$ and $\mathrm{PeV}$ anomalies," in Proceedings, Meeting of the APS Division of Particles and Fields (DPF 2015), Ann Arbor, MI, USA, August 2015.

[43] P. S. B. Dev, D. Kazanas, R. N. Mohapatra, V. L. Teplitz, and Y. Zhang, "Heavy right-handed neutrino dark matter and $\mathrm{PeV}$ neutrinos at IceCube," Journal of Cosmology and Astroparticle Physics, vol. 2016, no. 8, p. 34, 2016.

[44] Y. Bai, R. Lu, and J. Salvado, "Geometric compatibility of IceCube TeV-PeV neutrino excess and its galactic dark matter origin," Journal of High Energy Physics, vol. 1, p. 161, 2016.

[45] M. Ahlers, Y. Bai, V. Barger, and R. Lu, "Galactic neutrinos in the TeV to PeV range," Physical Review D, vol. 93, no. 1, article 013009, 2016.

[46] N. Hiroshima, R. Kitano, K. Kohri, and K. Murase, "Highenergy neutrinos from multi-body decaying dark matter," Physical Review D, vol. 97, no. 2, 2018.

[47] A. Esmaili and P. D. Serpico, "Are IceCube neutrinos unveiling PeV-scale decaying dark matter?," Journal of Cosmology and Astroparticle Physics, vol. 1311, p. 54, 2013.

[48] A. Esmaili and P. D. Serpico, "Gamma-ray bounds from EAS detectors and heavy decaying dark matter constraints," Journal of Cosmology and Astroparticle Physics, vol. 1510, p. 14, 2015.

[49] A. Bhattacharya, A. Esmaili, S. Palomares-Ruiz, and I. Sarcevic, "Probing decaying heavy dark matter with the 4year IceCube HESE data," Journal of Cosmology and Astroparticle Physics, vol. 2017, no. 7, p. 27, 2017.

[50] S. M. Boucenna, M. Chianese, G. Mangano et al., "Decaying leptophilic dark matter at IceCube," Journal of Cosmology and Astroparticle Physics, vol. 2015, p. 55, 2015.

[51] B. Dutta, Y. Gao, T. Li, C. Rott, and L. E. Strigari, "Leptoquark implication from the CMS and IceCube experiments," Physical Review D, vol. 91, no. 12, article 125015, 2015.

[52] U. K. Dey and S. Mohanty, "Constraints on leptoquark models from IceCube data," Journal of High Energy Physics, vol. 2016, no. 4, pp. 1-11, 2016.

[53] N. Mileo, A. de la Puente, and A. Szynkman, "Implications of a electroweak triplet scalar leptoquark on the ultra-high energy neutrino events at IceCube," Journal of High Energy Physics, vol. 2016, no. 11, p. 124, 2016.

[54] V. Barger, E. Basso, Y. Gao, and W.-Y. Keung, "Neutrino signals in IceCube from weak production of top and charm quarks," Physical Review D, vol. 95, no. 9, article 093002, 2017.

[55] B. Chauhan, B. Kindra, and A. Narang, "A Leptoquark explanation for $(g-2)_{\mu}, R_{K}, R_{K^{*}}$ and, IceCube PeV events," http:// arxiv.org/abs/1706.04598.
[56] L. A. Anchordoqui, C. A. Garcia Canal, H. Goldberg, D. Gomez Dumm, and F. Halzen, "Probing leptoquark production at IceCube," Physical Review D, vol. 74, no. 12, article 125021, 2006.

[57] J. D. Wells, "PeV-scale supersymmetry," Physical Review D, vol. 71, no. 1, article 015013, 2005.

[58] S. A. R. Ellis and J. D. Wells, "High-scale supersymmetry, the Higgs mass and gauge unification," http://arxiv.org/abs/1706 .00013 .

[59] Planck Collaborationhttp://arxiv.org/abs/1807.06211.

[60] Planck collaboration, "Planck 2015 results. XIII. Cosmological parameters," Astronomy \& Astrophysics, vol. 594, article A13, 2016.

[61] Planck collaboration, "Planck 2015 results. XX. Constraints on inflation," Astronomy \& Astrophysics, vol. 594, article A20, 2016

[62] Keck Array and bicep2 Collaborations, "Constraints on primordial gravitational waves using Planck, WMAP, and New BICEP2/Keck observations through the 2015 Season," Physical Review Letters, vol. 121, no. 22, article 221301, 2018.

[63] Keck Array and BICEP2 Collaborations, "Improved constraints on cosmology and foregrounds from BICEP2 and Keck array cosmic microwave background data with inclusion of $95 \mathrm{GHz}$ band," Physical Review Letters, vol. 116, article 031302, 2016.

[64] J. Ellis, D. V. Nanopoulos, and K. A. Olive, "No-scale supergravity realization of the Starobinsky model of inflation," Physical Review Letters, vol. 111, no. 11, article 111301, 2013.

[65] I. Garg and S. Mohanty, "No scale SUGRA SO(10) derived Starobinsky model of inflation," Physics Letters B, vol. 751, pp. 7-11, 2015.

[66] J. Ellis, M. A. G. Garcia, N. Nagata, D. V. Nanopoulos, and K. A. Olive, "Starobinsky-like inflation and neutrino masses in a no-scale $\mathrm{SO}(10)$ model," Journal of Cosmology and Astroparticle Physics, vol. 2016, no. 11, p. 18, 2016.

[67] J. Ellis, H. J. He, and Z. Z. Xianyu, "New Higgs inflation in a no-scale supersymmetric SU(5) GUT," Physical Review D, vol. 91, no. 2, article 021302, 2015.

[68] I. Garg and S. Mohanty, "No-scale SUGRA inflation and type-I seesaw," http://arxiv.org/abs/1711.01979.

[69] G. K. Chakravarty, G. Gupta, G. Lambiase, and S. Mohanty, "Plateau inflation in SUGRA-MSSM," Physics Letters B, vol. 760, pp. 263-266, 2016.

[70] G. K. Chakravarty, U. K. Dey, G. Lambiase, and S. Mohanty, "Plateau inflation in R-parity violating MSSM," Physics Letters $B$, vol. 763, pp. 501-506, 2016.

[71] L. Alvarez-Gaume, C. Gomez, and R. Jimenez, "Minimal inflation," Physics Letters B, vol. 690, no. 1, pp. 68-72, 2010.

[72] L. Alvarez-Gaume, C. Gomez, and R. Jimenez, "A minimal inflation scenario," Journal of Cosmology and Astroparticle Physics, vol. 1103, p. 27, 2011.

[73] G. K. Chakravarty and S. Mohanty, "Power law Starobinsky model of inflation from no-scale SUGRA," Physics Letters B, vol. 746, pp. 242-247, 2015

[74] S. Ferrara, R. Kallosh, and A. Linde, "Cosmology with nilpotent superfields," Journal of High Energy Physics, vol. 2014, no. 10, p. 143, 2014.

[75] G. K. Chakravarty, S. Das, G. Lambiase, and S. Mohanty, "Dilaton assisted two-field inflation from no-scale supergravity," Physical Review D, vol. 94, no. 2, article 023521, 2016. 
[76] S. Ferrara, R. Kallosh, A. Linde, and M. Porrati, "Minimal supergravity models of inflation," Physical Review D, vol. 88, no. 8, article $085038,2013$.

[77] F. Farakos, A. Kehagias, and A. Riotto, "On the Starobinsky model of inflation from supergravity," Nuclear Physics B, vol. 876, no. 1, pp. 187-200, 2013.

[78] S. Ferrara, P. Fre, and A. S. Sorin, "On the gauged Kähler isometry in minimal supergravity models of inflation," Fortschritte der Physik, vol. 62, no. 4, pp. 277-349, 2014.

[79] K. Nakayama, K. Saikawa, T. Terada, and M. Yamaguchi, "Structure of Kähler potential for D-term inflationary attractor models," Journal of High Energy Physics, vol. 2016, no. 5, p. $67,2016$.

[80] R. Deen, B. A. Ovrut, and A. Purves, "Supersymmetric sneutrino-Higgs inflation," Physics Letters B, vol. 762, pp. 441-446, 2016.

[81] K. Nakayama, F. Takahashi, and T. T. Yanagida, "Chaotic inflation with right-handed sneutrinos after Planck," Physics Letters B, vol. 730, pp. 24-29, 2014.

[82] C. S. Aulakh and I. Garg, "Supersymmetric seesaw inflation," Physical Review D, vol. 86, no. 6, article 065001, 2012.

[83] Y. G. Kim, H. M. Lee, and W.-I. Park, "The $\mu$ problem and sneutrino inflation," Journal of High Energy Physics, vol. 8, p. 126, 2011.

[84] C. Pallis and N. Toumbas, "Non-minimal sneutrino inflation, Peccei-Quinn phase transition and non-thermal leptogenesis," Journal of Cosmology and Astroparticle Physics, vol. 2011, no. 2, p. 19, 2011.

[85] S. Antusch, M. Bastero-Gil, J. P. Baumann, K. Dutta, S. F. King, and P. M. Kostka, "Gauge non-singlet inflation in SUSY GUTs,” Journal of High Energy Physics, vol. 8, p. 100, 2010.

[86] R. Allahverdi, K. Enqvist, J. Garcia-Bellido, and A. Mazumdar, "Gauge-invariant inflaton in the minimal supersymmetric standard model," Physical Review Letters, vol. 97, no. 19, article 191304, 2006.

[87] P. Athron, J.-h. Park, D. Stöckinger, and A. Voigt, "FlexibleSUSY-A spectrum generator generator for supersymmetric models," Computer Physics Communications, vol. 190, pp. 139-172, 2015.

[88] P. Athron, J.-h. Park, T. Steudtner, D. Stöckinger, and A. Voigt, "Precise Higgs mass calculations in (non-)minimal supersymmetry at both high and low scales," Journal of High Energy Physics, vol. 2017, no. 1, p. 79, 2017.

[89] F. Staub, "Exploring new models in all detail withSARAH," Advances in High Energy Physics, vol. 2015, Article ID 840780, 126 pages, 2015.

[90] F. Staub and W. Porod, "Improved predictions for intermediate and heavy supersymmetry in the MSSM and beyond," The European Physical Journal C, vol. 77, no. 5, p. 338, 2017.

[91] W. Porod, "SPheno, a program for calculating supersymmetric spectra, SUSY particle decays and SUSY particle production at e+e-colliders," Computer Physics Communications, vol. 153, no. 2, pp. 275-315, 2003.

[92] W. Porod and F. Staub, "SPheno 3.1: extensions including flavour, CP-phases and models beyond the MSSM," Computer Physics Communications, vol. 183, no. 11, pp. 2458-2469, 2012.

[93] G. Bélanger, F. Boudjema, P. Brun et al., "Indirect search for dark matter with micrOMEGAs_2.4," Computer Physics Communications, vol. 182, no. 3, pp. 842-856, 2011.
[94] G. Belanger, F. Boudjema, A. Pukhov, and A. Semenov, "micrOMEGAs_3: A program for calculating dark matter observables," Computer Physics Communications, vol. 185, no. 3, pp. 960-985, 2014.

[95] M. Carena, D. Choudhury, S. Lola, and C. Quigg, "Manifestations ofR-parity violation in ultrahigh-energy neutrino interactions," Physical Review D, vol. 58, no. 9, article 095003, 1998.

[96] P. S. B. Dev, D. K. Ghosh, and W. Rodejohann, "R-parity violating supersymmetry at IceCube," Physics Letters B, vol. 762, pp. 116-123, 2016.

[97] D. Bečirević, B. Panes, O. Sumensari, and R. Z. Funchal, "Seeking leptoquarks in IceCube," Journal of High Energy Physics, vol. 2018, no. 6, p. 32, 2018.

[98] S. Ando and K. Ishiwata, "Constraints on decaying dark matter from the extragalactic gamma-ray background," Journal of Cosmology and Astroparticle Physics, vol. 2015, no. 5, p. 24, 2015.

[99] S. Shirai, F. Takahashi, and T. T. Yanagida, "R-violating decay of wino dark matter and electron/positron excesses in the PAMELA/Fermi experiments," Physics Letters B, vol. 680, no. 5, pp. 485-488, 2009.

[100] K. Hamaguchi, T. Moroi, and K. Nakayama, "AMS-02 antiprotons from annihilating or decaying dark matter," Physics Letters B, vol. 747, pp. 523-528, 2015.

[101] H. Baer, A. Mustafayev, E.-K. Park, and X. Tata, "Collider signals and neutralino dark matter detection in relic-densityconsistent models without universality," Journal of High Energy Physics, vol. 2008, no. 5, p. 58, 2008.

[102] S. Akula, M. Liu, P. Nath, and G. Peim, "Naturalness, supersymmetry and implications for LHC and dark matter," Physics Letters B, vol. 709, no. 3, pp. 192-199, 2012.

[103] N. Bhattacharyya, A. Choudhury, and A. Datta, "Low mass neutralino dark matter in minimal supergravity and more general models in the light of LHC data," Physical Review $D$, vol. 84, no. 9, article 095006, 2011.

[104] H. Baer, V. Barger, and A. Mustafayev, "Neutralino dark matter in mSUGRA/CMSSM with a $125 \mathrm{GeV}$ light Higgs scalar," Journal of High Energy Physics, vol. 2012, no. 5, p. 91,2012

[105] P. S. Bhupal Dev, S. Mondal, B. Mukhopadhyaya, and S. Roy, "Phenomenology of light sneutrino dark matter in cMSSM/mSUGRA with inverse seesaw," Journal of High Energy Physics, vol. 9, p. 110, 2012.

[106] B. Kaufman, P. Nath, B. D. Nelson, and A. B. Spisak, "Light stops and observation of supersymmetry at LHC run II," Physical Review D, vol. 92, no. 9, article 095021, 2015.

[107] D. Hu, T. Li, A. Lux, J. A. Maxin, and D. V. Nanopoulos, "General no-scale supergravity: an F-SU(5) Tale," Physics Letters B, vol. 771, pp. 264-270, 2017.

[108] H. Baer, C. Balazs, A. Belyaev, T. Krupovnickas, and X. Tata, "Updated reach of CERN LHC and constraints from relic density, $\mathrm{b} \rightarrow \mathrm{s} \gamma$ and a $\mu$ in the mSUGRA model," Journal of High Energy Physics, vol. 2003, no. 6, p. 54, 2003.

[109] H. P. Nilles, "Supersymmetry, supergravity and particle physics," Physics Reports, vol. 110, no. 1-2, pp. 1-162, 1984.

[110] I. Niessen, "Supersymmetric phenomenology in the mSUGRA parameter space," Ph.D. thesis, Nijmegen U., IMAPP, 2008.

[111] U. Chattopadhyay, A. Corsetti, and P. Nath, "WMAP constraints, supersymmetric dark matter, and implications for 
the direct detection of supersymmetry," Physical Review D, vol. 68, no. 3, article 035005, 2003.

[112] K. L. Chan, U. Chattopadhyay, and P. Nath, "Naturalness, weak scale supersymmetry, and the prospect for the observation of supersymmetry at the Fermilab Tevatron and at the CERN LHC," Physical Review D, vol. 58, no. 9, article 096004, 1998.

[113] H. Baer, V. Barger, and M. Savoy, "Supergravity gauge theories strike back: there is no crisis for SUSY but a new collider may be required for discovery," Physica Scripta, vol. 90, no. 6, article 068003, 2015.

[114] G. Coughlan, W. Fischler, E. W. Kolb, S. Raby, and G. G. Ross, "Cosmological problems for the Polonyi potential," Physics Letters B, vol. 131, no. 1-3, pp. 59-64, 1983.

[115] J. R. Ellis, D. V. Nanopoulos, and M. Quiros, "On the axion, dilaton, Polonyi, gravitino and shadow matter problems in supergravity and superstring models," Physics Letters B, vol. 174, no. 2, pp. 176-182, 1986.

[116] T. Banks, D. B. Kaplan, and A. E. Nelson, "Cosmological implications of dynamical supersymmetry breaking," Physical Review D, vol. 49, no. 2, pp. 779-787, 1994.

[117] B. de Carlos, J. Casas, F. Quevedo, and E. Roulet, "Modelindependent properties and cosmological implications of the dilaton and moduli sectors of $4 \mathrm{D}$ strings," Physics Letters B, vol. 318, no. 3, pp. 447-456, 1993.

[118] A. Linde, Y. Mambrini, and K. A. Olive, "Supersymmetry breaking due to moduli stabilization in string theory," Physical Review D, vol. 85, no. 6, article 066005, 2012.

[119] E. Dudas, A. Linde, Y. Mambrini, A. Mustafayev, and K. A. Olive, "Strong moduli stabilization and phenomenology," The European Physical Journal C, vol. 73, no. 1, article 2268, 2013.

[120] S. K. Soni and H. A. Weldon, "Analysis of the supersymmetry breaking induced by $\mathrm{N}=1$ supergravity theories," Physics Letters B, vol. 126, no. 3-4, pp. 215-219, 1983.

[121] V. S. Kaplunovsky and J. Louis, "Model-independent analysis of soft terms in effective supergravity and in string theory," Physics Letters B, vol. 306, no. 3-4, pp. 269-275, 1993.

[122] E. Dudas and S. K. Vempati, "Large D-terms, hierarchical soft spectra and moduli stabilisation," Nuclear Physics B, vol. 727, no. 1-2, pp. 139-162, 2005.

[123] A. Brignole, L. E. Ibanez, and C. Munoz, "Towards a theory of soft terms for the supersymmetric standard model," Nuclear Physics B, vol. 422, no. 1-2, pp. 125-171, 1994.

[124] A. Brignole, L. E. Ibanez, and C. Munoz, "Soft supersymmetry breaking terms from supergravity and superstring models," Advanced Series on Directions in High Energy Physics, vol. 18, pp. 125-148, 1998.

[125] K. Griest and M. Kamionkowski, "Unitarity limits on the mass and radius of dark-matter particles," Physical Review Letters, vol. 64, no. 6, pp. 615-618, 1990.

[126] C. Rott, K. Kohri, and S. C. Park, "Superheavy dark matter and IceCube neutrino signals: bounds on decaying dark matter," Physical Review D, vol. 92, no. 2, article 023529, 2015.

[127] M. Re Fiorentin, V. Niro, and N. Fornengo, "A consistent model for leptogenesis, dark matter and the IceCube signal," Journal of High Energy Physics, vol. 2016, no. 11, p. 22, 2016.

[128] B. Audren, J. Lesgourgues, G. Mangano, P. D. Serpico, and T. Tram, "Strongest model-independent bound on the lifetime of dark matter," Journal of Cosmology and Astroparticle Physics, vol. 2014, no. 12, p. 28, 2014.
[129] K. Garrett and G. Duda, "Dark matter: a primer," Advances in Astronomy, vol. 2011, Article ID 968283, 22 pages, 2011.

[130] H. Murayama, "Physics beyond the standard model and dark matter," in Les Houches Summer School - Session 86: Particle Physics and Cosmology: The Fabric of Spacetime Les Houches, France, July-August 2006https://inspirehep.net/record/ 748786/files/arXiv:0704.2276.pdf.

[131] E. W. Kolb and M. S. Turner, The Early Universe, PublisherAddison-Wesley Publishing Company, 1989.

[132] Planck collaboration, "Planck 2013 results. XVI. Cosmological parameters," Astronomy \& Astrophysics, vol. 571, article A16, 2014

[133] Z. Berezhiani, A. D. Dolgov, and I. I. Tkachev, "Reconciling Planck results with low redshift astronomical measurements," Physical Review D, vol. 92, no. 6, article 061303, 2015.

[134] E. A. Baltz and P. Gondolo, "Neutralino decay rates with explicitR-parity violation," Physical Review D, vol. 57, no. 5, pp. 2969-2973, 1998.

[135] S. Palomares-Ruiz, A. C. Vincent, and O. Mena, "Spectral analysis of the high-energy IceCube neutrinos," Physical Review D, vol. 91, no. 10, article 103008, 2015.

[136] IceCube collaboration, "Atmospheric and astrophysical neutrinos above $1 \mathrm{TeV}$ interacting in IceCube," Physical Review $D$, vol. 91, article 022001, 2015.

[137] A. Esmaili, A. Ibarra, and O. L. G. Peres, "Probing the stability of superheavy dark matter particles with high-energy neutrinos," Journal of Cosmology and Astroparticle Physics, vol. 2012, no. 11, p. 34, 2012. 